\title{
Solutions and Thermodynamics of Charged Rotating Black Holes in a Fuzzy Space
}

\author{
Abderrahman El Boukili, ${ }^{1}$ Mourad Nach, ${ }^{1}$ Hamid Chaqsare, ${ }^{1}$ \\ Moulay Driss Aouragh, ${ }^{2}$ and Moulay Brahim Sedra ${ }^{1}$ \\ ${ }^{1}$ Laboratoire des Hautes Énergies, Sciences de l'Ingénierie et Réacteurs, Université Ibn Tofail, Faculté des Sciences, \\ Département de Physique, 14000 Kénitra, Morocco \\ ${ }^{2}$ Faculté des Sciences et Techniques, Université Moulay Ismail, 52000 Errachidia, Morocco
}

Correspondence should be addressed to Abderrahman EL Boukili; aelboukili@gmail.com

Received 24 April 2013; Accepted 28 May 2013

Academic Editors: A. Belhaj, C. A. D. S. Pires, and A. Koshelev

Copyright (C) 2013 Abderrahman EL Boukili et al. This is an open access article distributed under the Creative Commons Attribution License, which permits unrestricted use, distribution, and reproduction in any medium, provided the original work is properly cited.

We investigate the effect of fuzzy space on black hole and its thermodynamic Properties; we also present our results in different types of black holes according to the parameters $Q$ and $J$ representing electric and magnetic charges.

\section{Introduction}

In general relativity, there exist three classes of black holes, namely, Schwarzschild black hole, Kerr black hole and Reissner-Nordström black hole, or Kerr-Newman black hole [1]. In fact, the solutions of Einstein's field equation for the gravitational field of an electrically charged point mass were obtained in 1918 by Nordström and Reissner [2, 3], not long after Karl Schwarzschild had found the Schwarzschild metric as a solution for a point mass without electric charge and angular momentum [4].

However, the cosmic behavior of black holes has been studied in different points such as the cosmic history of black hole [5], the dynamical evolution and observational signatures of massive black hole [6].

In this paper we will examine the effect of fuzzy space [7] on a static, charged, and rotating black hole. We will investigate thermodynamic properties such as Hawking temperature and entropy.

This paper is organized as follows. In Section 2, we present Schwarzschild black hole in fuzzy space. In Section 3, we present the electric charged black hole in fuzzy space. We study a magnetic fuzzy black hole in Section 4 . In Section 5, we give the combined case where $J>0$ and $Q>0$. We give some thermodynamic properties of fuzzy black hole in diverse cases in Section 6. Finally, we discuss our results in Section 7.

\section{Fuzzy Schwarzschild Black Hole}

We consider Schwarzschild black hole in a fuzzy space with the mass density of point particle described by [8]

$$
\rho_{h}(r)=\frac{1}{2 \pi h^{2}} \exp \left(-\frac{r}{h}\right) .
$$

The covariant conservation law $\nabla_{\mu} T^{\mu \nu}$ and the condition of the metric coefficient $g_{00}=-g_{r r}^{-1}$ for the noncommutative Schwarzschild-like metric and the energy momentum tensor are given by

$$
T_{\mu}^{v}=\operatorname{Diag}\left(-\rho_{h}(r), p_{r}(r), p_{\perp}(r), p_{\perp}(r)\right),
$$

where

$$
p_{r}=-\rho_{h}, \quad p_{\perp}=p_{r}-\frac{r}{2} \partial_{r} \rho_{h} .
$$

The asymptotic solution of Einstein's equation (2), using (1) as the matter source, is the same as replacing the mass of 
Dirac delta function source in Schwarzschild spacetime by the effective mass of smeared source [9]

$$
\begin{aligned}
M_{h}(r) & =M \int d^{3} x \rho_{h}(r)=4 \pi \int_{0}^{r} r^{\prime 2} \rho_{h}\left(r^{\prime}\right) d r^{\prime} \\
& =M\left[1-\left(\frac{1}{2}\left(\frac{r}{h}\right)^{2}+\frac{r}{h}+1\right) \exp \left(-\frac{r}{h}\right)\right] .
\end{aligned}
$$

The geometry of fuzzy black hole is described by the line element

$$
\begin{aligned}
d S^{2}= & -\left(1-\frac{2 M_{h}(r)}{r}\right) d t^{2}+\left(1-\frac{2 M_{h}(r)}{r}\right) d r^{2} \\
& +r^{2}\left(d \theta^{2}+\sin ^{2} \theta d \phi^{2}\right),
\end{aligned}
$$

where

$$
g_{00}(r)=g^{r r}(r)=f(r)=1-\frac{2 M_{h}(r)}{r} .
$$

The event horizon of the black hole is given by setting $f\left(r_{h}\right)=$ 0 , and the nonzero solution of this equation satisfies

$$
r_{h}=2 M_{h}(r)=2 M\left[1-\left(\frac{1}{2}\left(\frac{r_{h}}{h}\right)^{2}+\frac{r_{h}}{h}+1\right) \exp \left(-\frac{r_{h}}{h}\right)\right]
$$

Instead of a single-event horizon, there are different possibilities: (i) we have two distinct horizons for $M>M_{0}$, (ii) in this case, we have one degenerate horizon (external black hole) for $M=M_{0}$, and (iii) no horizon for $M<M_{0}$.

\section{Charged Black Hole in a Fuzzy Space}

In this case, we have a black hole with $J=0$ and $Q>0$. As mentioned in [10], the geometry of a fuzzy black hole is described by the line element

$$
d S^{2}=-f(r) d t^{2}+\frac{1}{f(r)} d r^{2}+r^{2} d \phi^{2}
$$

Its Einstein's field equations are written accordingly as

$$
\begin{gathered}
\frac{1}{r} \frac{d f(r)}{d r}=-32 \pi M \rho_{h}(r)-\frac{1}{2} E^{2}, \\
\frac{d^{2} f(r)}{d r^{2}}=32 \pi p_{\perp}(r)+\frac{1}{2} E^{2} .
\end{gathered}
$$

Solving the previous equations, we find

$$
\begin{gathered}
f(r)=-8 M\left[1-\left(\frac{1}{2}\left(\frac{r}{h}\right)^{2}+\frac{r}{h}+1\right) \exp \left(-\frac{r}{h}\right)\right]+\frac{r^{2}}{l^{2}} \\
-\frac{Q^{2}}{8 \pi^{2}}\left[\ln r+2 e^{-(r / h)}-\left(\frac{2 r}{h}+\frac{5}{4}\right) e^{-2(r / h)}\right. \\
\left.-2 \int \frac{e^{-(r / h)}}{r} d r+\int \frac{e^{-2(r / h)}}{r} d r\right],
\end{gathered}
$$

where $\operatorname{Ei}(z)$ represents the exponential integral function defined as

$$
\operatorname{Ei}(z)=-\int_{-z}^{\infty} \frac{e^{-t}}{t} d t
$$

and a smeared distribution function is described by

$$
\rho_{h}(r)=\frac{1}{2 \pi h^{2}} \exp \left(-\frac{r}{h}\right), \quad 2 \mathrm{D} \text { fuzzy space. }
$$

In this case, the metric $f(r)$ takes the following form:

$$
\begin{gathered}
f(r)=-8 M\left[1-\left(\frac{1}{2}\left(\frac{r}{h}\right)^{2}+\frac{r}{h}+1\right) \exp \left(-\frac{r}{h}\right)\right]+\frac{r^{2}}{l^{2}} \\
-\frac{Q^{2}}{8 \pi^{2}}\left[\ln r-2 h \operatorname{Ei}\left(-\frac{r}{h}\right)+\frac{h}{2} \operatorname{Ei}\left(-2 \frac{r}{h}\right)\right. \\
\left.+2 e^{-(r / h)}-2 \frac{r}{h} e^{-(2 r / h)}-\frac{5}{4} e^{-(2 r / h)}\right] .
\end{gathered}
$$

Note that when $r / h \rightarrow \infty$, either when considering a large black hole $(r \rightarrow \infty)$ or the commutative limit $(h \rightarrow 0)$, we obtain the following metric which defines charged BTZ solution [11]:

$$
f(r)=-8 M+\frac{r^{2}}{l^{2}}-\frac{Q^{2}}{8 \pi^{2}} \ln r
$$

The metric (13) describes the geometry of a fuzzy black hole with the corresponding event horizon given by the following condition imposed on $f(r)$. Equation (13) cannot be solved in closed form. However, by plotting $f(r)$, one can see obvious intersections with the $r$-axis and determine numerically the existence of horizons and their radii.

In view of these results, there can be no black hole if the original mass is less than the lower limit mass $M_{0}$. The horizon of the external black hole is determined by the conditions $f=0$ and $\partial_{r} f=0$, and the mass of the external black hole can be written as

$$
\begin{aligned}
M_{0}= & \frac{h^{3}}{2 l^{2}} e^{r_{0} / h}-\frac{Q^{2} h^{3}}{32 \pi^{2} r_{0}} \\
& \times\left[\frac{1}{r_{0}} e^{r_{0} / h}+2\left(\frac{h}{r_{0}}-\frac{1}{h}\right)\right. \\
& \left.+\left(\frac{4 r_{0}}{h^{2}}-\frac{h^{2}}{4 r_{0}}+\frac{1}{2 h}\right) e^{-\left(r_{0} / h\right)}\right] .
\end{aligned}
$$

\section{Rotating Fuzzy Black Hole}

The metric of a fuzzy black hole (with $J>0$ and $Q=0$ ) is given in $[12,13]$ by

$$
d S^{2}=-f(r) d t^{2}+\frac{1}{f(r)} d r^{2}+r^{2} d \phi^{2},
$$




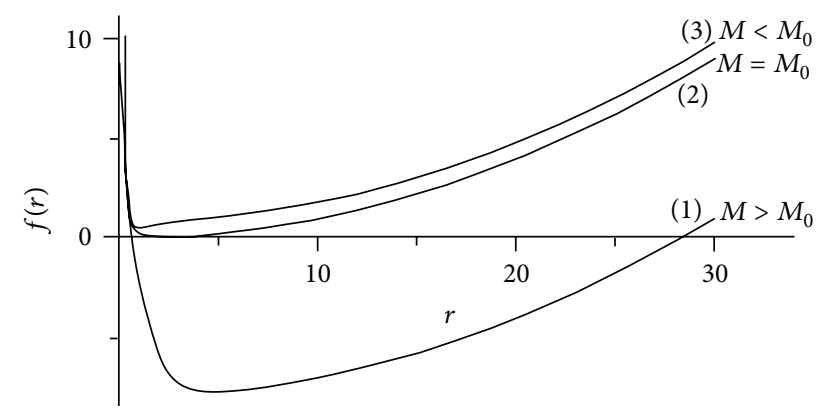

FIgURE 1: Metric function $f$ as a function of $r$. We have taken the values $\left(h=0.1, l=10, J=1\right.$, and $\left.M_{0} \approx 0.001\right)$.

where

$$
f(r)=-8 M\left[1-\left(\frac{1}{2}\left(\frac{r}{h}\right)^{2}+\frac{r}{h}+1\right) e^{-(r / h)}\right]+\frac{r^{2}}{l^{2}}+\frac{J^{2}}{4 r^{2}} .
$$

Note that when $h \rightarrow 0$, we obtain the well-known BTZ rotating solution with angular momentum $J$ and total mass $M, f(r)=-8 M+\left(r^{2} / l^{2}\right)+\left(J^{2} / 4 r^{2}\right)$. The metric (16) describes the geometry of a fuzzy black hole with the corresponding event horizon given by the following condition imposed on $f(r)$ :

$$
\begin{aligned}
f(r) & =-8 M\left[1-\left(\frac{1}{2}\left(\frac{r_{ \pm}}{h}\right)^{2}+\frac{r_{ \pm}}{h}+1\right) e^{r_{ \pm} / h}\right]+\frac{r_{ \pm}^{2}}{l^{2}}+\frac{J^{2}}{4 r_{ \pm}^{2}} \\
& =0 .
\end{aligned}
$$

This equation cannot be solved in closed form. However, by plotting $f(r)$, one can read intersections with the $r$-axis and determine numerically the existence of horizon(s) and their radii.

Figure 1 shows that the existence of angular momentum introduces new behavior with respect to the noncommutative black hole studied by Myung and Yoon [14]. Instead of a single-event horizon, there are different possibilities: we have two distinct horizons for $M>M_{0}$, we have one degenerate horizon (external black hole) for $M=M_{0}$, and no horizon for $M<M_{0}$.

In view of these results, there can be no black hole if the original mass is less than the lower limit mass $M_{0}$. The horizon of the external black hole is determined by the conditions $f=0$ and $\partial_{r} f=0$, which gives

$$
\begin{aligned}
4 r^{4} & -4 r^{4}\left(\frac{1}{2}\left(\frac{r}{h}\right)^{2}+\frac{r}{h}+1\right) e^{-r / h}-r e^{-(r / h)} \\
& =\frac{J^{2} l^{2}}{4}\left(r e^{-(r / h)}+4-\left(2\left(\frac{r}{h}\right)^{2}+4 \frac{r}{h}+4\right) e^{-(r / h)}\right),
\end{aligned}
$$

and then, the mass of the extremal black hole can be written as

$$
\begin{aligned}
M_{0}= & \left(\frac{r_{0}^{2}}{8 l^{2}}+\frac{J^{2}}{32 r_{0}^{2}}\right) \\
& \times\left[1-\left(\frac{1}{2}\left(\frac{r_{0}^{2}}{h}\right)+\frac{r_{0}}{h}+1\right) \exp \left(-\frac{r_{0}}{h}\right)\right]^{-1} .
\end{aligned}
$$

In the commutative limit $h \rightarrow 0$, the extreme black hole has the horizon at

$$
r_{0}^{\mathrm{BTZ}}=\sqrt{\frac{J l}{2}}
$$

and its mass is

$$
M_{0}^{\mathrm{BTZ}}=\frac{1}{8} \frac{J}{l}
$$

\section{Charged Rotating Fuzzy Black Hole}

In this section, we will combine our results for the case of an electric and magnetic charged black hole; such type is known as Kerr-Newman black hole $(J>0$ and $Q>0)$. Its metric is given by

$$
\begin{gathered}
d S^{2}=-f(r) d t^{2}+\frac{1}{f(r)} d r^{2}+r^{2} d \phi^{2}, \\
f(r)=-8 M+\frac{r^{2}}{l^{2}}-\frac{Q^{2}}{8 \pi^{2}} \ln r+\frac{J^{2}}{4 r^{2}} .
\end{gathered}
$$

The metric (23) describes the geometry of a fuzzy black hole with the corresponding event horizon given by the following condition imposed on $f(r)$ :

$$
\begin{array}{r}
f(r)=-8 M\left[1-\left(\frac{1}{2}\left(\frac{r}{h}\right)^{2}+\frac{r}{h}+1\right) \exp \left(-\frac{r}{h}\right)\right]+\frac{r^{2}}{l^{2}} \\
+\frac{J^{2}}{4 r^{2}}-\frac{Q^{2}}{8 \pi^{2}}\left[\ln r-2 h \operatorname{Ei}\left(-\frac{r}{h}\right)+\frac{h}{2} \operatorname{Ei}\left(-2 \frac{r}{h}\right)\right. \\
+\exp \left(-\frac{r}{h}\right)-2 \frac{r}{h} \exp \left(-\frac{2 r}{h}\right) \\
\left.-\frac{5}{4} \exp \left(-\frac{2 r}{h}\right)\right] .
\end{array}
$$

The derivative of the metric function is given by

$$
\begin{aligned}
\partial_{r} f=-\frac{8 M}{h^{3}} & \exp \left(-\frac{r}{h}\right)+\frac{2 r}{l^{2}}-\frac{J^{2}}{2 r^{3}} \\
-\frac{Q^{2}}{8 \pi^{2} r}[1 & +2 \exp \left(-\frac{r}{h}\right)-\exp \left(-2 \frac{r}{h}\right) \\
& -2 \frac{r}{h} \exp \left(-\frac{r}{h}\right)-2 \frac{r}{h} \exp \left(-2 \frac{r}{h}\right) \\
& \left.\times \frac{4 r^{2}}{h} \exp \left(-2 \frac{r}{h}\right)-\frac{5 r}{2 h} \exp \left(-2 \frac{r}{h}\right)\right] .
\end{aligned}
$$


In view of these results, there can be no black hole if the original mass is less than the lower limit mass $M_{0}$. The horizon of the external black hole is determined by the conditions $f=0$ and $\partial_{r} f=0$, which gives

$$
\begin{aligned}
& r^{2} \exp \left(-\frac{r}{h}\right)-4 r h^{3}\left(1-\lambda(r, h) \exp \left(-\frac{r}{h}\right)\right) \\
& =-\frac{J^{2} l^{2}}{4 r^{2}} \exp \left(-\frac{r}{h}\right) \\
& \times\left(e^{-(r / h)}+\frac{4 h^{3}}{r}\left(1-\lambda(r, h) \exp \left(-\frac{r}{h}\right)\right)\right) \\
& -\frac{Q^{2} l^{2}}{8 \pi^{2}}\left\{r \operatorname { e x p } ( - \frac { r } { h } ) \left[\ln r+2 \operatorname{Ei}\left(-\frac{r}{h}\right)-\operatorname{Ei}\left(-2 \frac{r}{h}\right)\right.\right. \\
& \quad+2 \exp \left(-\frac{r}{h}\right)-2 \frac{r}{h} \exp \left(-\frac{2 r}{h}\right) \\
& +2 h^{3}\left(1-\lambda(r, h) \exp \left(-\frac{2 r}{h}\right)\right] \\
& \times\left(1+2 \exp \left(-\frac{r}{h}\right)-\exp \left(-\frac{2 r}{h}\right)\right) \\
& +\frac{2}{h} r \exp \left(-\frac{r}{h}\right)-\left(\frac{h}{2}+\frac{5}{2 h}\right) r \exp \left(-\frac{2 r}{h}\right) \\
& \left.+r^{2} \exp \left(-\frac{2 r}{h}\right)\right\},
\end{aligned}
$$

where $\lambda(r, h)=(1 / 2)(r / h)^{2}+(r / h)+1$, and then, the mass of the external black hole can be written as

$$
\begin{aligned}
& M_{0} \\
& =4 h^{3} \exp \left(-\frac{r_{0}}{h}\right) \\
& \quad \times\left\{\frac{2 r_{0}}{h}-\frac{J^{2}}{2 r_{0}^{3}}-\frac{Q^{2}}{8 \pi^{2} r_{0}}\right. \\
& \quad \times\left[1+2 \exp \left(-\frac{r_{0}}{h}\right)-\exp \left(-2 \frac{r_{0}}{h}\right)\right. \\
& \quad-\frac{2}{h} r_{0} \exp \left(-\frac{r_{0}}{h}\right)-\left(\frac{h}{2}+\frac{5}{2 h}\right) r_{0} \exp \left(-2 \frac{r_{0}}{h}\right) \\
& \left.\left.\quad+r_{0}^{2} \exp \left(-2 \frac{r_{0}}{h}\right)\right]\right\} .
\end{aligned}
$$

\section{Thermodynamic Properties}

6.1. Static Black Hole. The Hawking temperature is calculated by $[15,16]$

$$
\begin{aligned}
T_{H}= & \left(\frac{1}{4 \pi} \frac{d g_{00}}{d r}\right)_{r=r_{h}}=\frac{1}{4 \pi r_{h}} \\
& \times\left[1-\frac{(1 / 2)\left(r_{h} / h\right)^{3} \exp \left(-\left(r_{h} / h\right)\right)}{1-\left((1 / 2)\left(r_{h} / h\right)^{2}+\left(r_{h} / h\right)+1\right) \exp \left(-\left(r_{h} / h\right)\right)}\right] .
\end{aligned}
$$

6.2. Charged Black Hole. Hawking temperature [17] is as follows:

$$
\begin{aligned}
T_{H}=\frac{1}{4 \pi}\left|\frac{\partial f(r)}{\partial r}\right|_{r=r_{+}} & \\
=\frac{1}{4 \pi} \mid & -\frac{4 M_{H} r_{+}^{2}}{h^{3}} e^{-\left(r_{+} / h\right)}+2 \frac{r_{+}}{l^{2}} \\
& -\frac{Q^{2}}{8 \pi^{2}}\left[\frac{1}{r_{+}}+2\left(\frac{h^{2}}{r_{+}}-\frac{1}{h}\right) e^{-\left(r_{+} / h\right)}\right. \\
& \left.+\left(\frac{4 r_{+}}{h^{2}}+\frac{1}{2 h}-\frac{h^{2}}{4 r_{+}}\right) e^{-\left(2 r_{+} / h\right)}\right] \mid,
\end{aligned}
$$

where

$$
\begin{aligned}
M_{H}= & \left(8 \pi^{2} r_{+}^{2}-Q^{2} l^{2}\right. \\
\times & {\left[\ln r_{+}-2 h \operatorname{Ei}\left(-\frac{r_{+}}{h}\right)+\frac{h}{2} \operatorname{Ei}\left(-2 \frac{r_{+}}{h}\right)\right.} \\
& \left.\left.+2 e^{-\left(r_{+} / h\right)}-2 \frac{r_{+}}{h} e^{-\left(r_{+} / h\right)}-\frac{5}{4} e^{-2\left(r_{+} / h\right)}\right]\right) \\
\times & \left(64 \pi^{2} l^{2}\left[1-\left(\frac{1}{2}\left(\frac{r_{+}}{h}\right)^{2}+\frac{r_{+}}{h}+1\right) \exp \left(-\frac{r_{+}}{h}\right)\right]\right)^{-1} .
\end{aligned}
$$

The first law of thermodynamics for a charged black hole form is

$$
d M=T_{H} d S+\Phi d Q,
$$

where the electrostatic potential is given by

$$
\begin{gathered}
\Phi=\left(\frac{\partial M}{\partial Q}\right)_{r_{+}} \\
=\frac{-Q}{32 \pi^{2}}\left(\ln r_{+}-2 h \operatorname{Ei}\left(-\frac{r_{+}}{h}\right)+\frac{h}{2} \operatorname{Ei}\left(-\frac{2 r_{+}}{h}\right)\right. \\
\left.\quad+2 e^{-\left(r_{+} / h\right)}-2 \frac{r_{+}}{h} e^{-2\left(r_{+} / h\right)}-\frac{5}{4} e^{-\left(2 r_{+} / h\right)}\right) \\
\quad \times\left(1-\left(\frac{1}{2}\left(\frac{r_{+}^{2}}{h}\right)+\frac{r_{+}}{h}+1\right) \exp \left(-\frac{r_{+}}{h}\right)\right)^{-1} .
\end{gathered}
$$


We calculate the entropy as

$$
S=\int_{r_{0}}^{r_{+}} \frac{1}{T_{H}} d M,
$$

which gives

$$
\begin{aligned}
S & =\frac{\pi}{2} \int_{r_{0}}^{r_{+}} \frac{1}{1-\exp (-(\xi / h))} d \xi \\
& =\frac{\pi h}{2} \ln \left(\frac{\exp \left(-\left(r_{+} / h\right)\right)-1}{\exp \left(r_{0} / h\right)-1}\right) .
\end{aligned}
$$

6.3. Rotating Black Hole. For rotating fuzzy black hole, the hawking temperature of the geometry of the fuzzy black hole is given by [18]

$$
\begin{aligned}
T_{H}= & \frac{1}{4 \pi}\left|\frac{\partial f(r)}{\partial r}\right|_{r=r_{+}} \\
= & \frac{1}{2 \pi} \frac{r_{+}}{l^{2}} \\
& \times\left[1-\frac{J^{2} l^{2}}{4 r_{+}^{2}}\right. \\
& \left.\quad+\frac{r_{+}^{3}+\left(J^{2} l^{2} / 4 r_{+}\right)}{4 h^{3}\left[1+\left(r_{+} / h\right)+(1 / 2)\left(r_{+} / h\right)^{2}-e^{-\left(r_{+} / h\right)}\right]}\right],
\end{aligned}
$$

where

$$
\begin{aligned}
M_{H}= & \left(\frac{r_{+}^{2}}{8 l^{2}}+\frac{J^{2}}{32 r_{+}^{2}}\right) \\
& \times\left(1-\left(\frac{1}{2}\left(\frac{r_{+}}{h}\right)^{2}+\frac{r_{+}}{h}+1\right) e^{-\left(r_{+} / h\right)}\right)^{-1} .
\end{aligned}
$$

The temperature is a monotonically increasing function towards the horizon radius for large black holes, and the temperature of the extreme black hole is zero.

For large black holes, that is, $\left(r_{+} / h\right) \gg 0$, one recovers the temperature of the rotating BTZ black hole

$$
T_{H}^{\mathrm{BTZ}}=\frac{1}{2 \pi} \frac{r_{+}}{l^{2}}\left[1-\frac{J^{2} l^{2}}{4 r_{+}^{4}}\right] .
$$

The first law of thermodynamics for a charged black hole form is

$$
d M=T_{H} d S+\Phi d Q
$$

where the angular velocity of the black hole is given by

$$
\Phi=\left(\frac{\partial M}{\partial J}\right)_{r_{+}}=\frac{J^{2}}{2 r_{+}^{2}} .
$$

It is the same rotating BTZ solution. Equation (37) can be written in the Gibbs form as $[10,12]$

$$
d S=\frac{1}{T} d M-\frac{\Phi}{T} d J
$$

At the outer horizon, $r=r_{+}$, we have $M=M\left(r_{+}, J\right)$ and

$$
d M=\frac{\partial M}{\partial r_{+}} d r_{+}+\Phi d J .
$$

Using (39) we get

$$
d M=\frac{1}{T} \frac{\partial M}{\partial r_{+}} d r_{+}
$$

where $S$ is the entropy of the noncommutative black hole. From (20) we finally obtain

$$
S=\int_{r_{0}}^{r_{+}} \frac{1}{1-e^{-(\xi / h)}} d \xi,
$$

where the entropy is a function of $r_{+}$. Note that, in the large black hole limit, the entropy function corresponds to the Bekenstein-Hawking entropy (area law), $S_{\mathrm{BH}}=\pi r_{+} / 2$, for the rotating BTZ geometry.

\section{Conclusion}

In this paper, we have constructed a static, electric, and magnetic (rotating) black hole in a fuzzy space by using an anisotropic perfect fluid fuzzy black hole and a smeared distribution function as an electric and magnetic charge. We have investigated the Hawking temperature and entropy.

We have also studied the combined case where both magnetic and electric charges of the black holes in a fuzzy space exist.

Note that when $r / h \rightarrow \infty$, either when considering a large black hole $(r \rightarrow \infty)$ or the commutative limit $(h \rightarrow$ 0 ), we obtain the metric of charged BTZ solutions with the electric charge $Q$ and total mass $M$

$$
f(r)=-8 M+\frac{r^{2}}{l^{2}}-\frac{Q^{2}}{8 \pi^{2}} \ln r,
$$

and we obtain the well-known BTZ rotating solution with angular momentum $J$ and the total mass $M$

$$
f(r)=-8 M+\frac{r^{2}}{l^{2}}+\frac{J^{2}}{4 r^{2}} .
$$

We have obtained similar results in the case of a black hole in Moyal space, namely, the existence of the event horizon and the Hawking temperature and entropy $[10,12]$.

\section{References}

[1] S. D. Mathur, "The quantum structure of black holes," Classical and Quantum Gravity, vol. 23, no. 11, pp. R115-R168, 2006.

[2] G. Nordström, "On the energy of the gravitational field in Einstein's theory," Koninklijke Nederlandse Akademie Van Wetenschappen, vol. 20, pp. 1238-1245, 1918.

[3] H. Reissner, "Uber die Eigengravitation des elektrischen Feldes nach der Einsteins chen Theorie," Annalen Der Physik, vol. 355, no. 9, pp. 106-120.

[4] M. D. Kruskal, "Maximal extension of Schwarzschild metric," Physical Review, vol. 119, pp. 1743-1745, 1960. 
[5] E. Treister and C. M. Urry, "The cosmic history of black hole growth from deep multiwavelength surveys," Advances in Astronomy, vol. 2012, Article ID 516193, 21 pages, 2012.

[6] M. Dotti, A. Sesana, and R. Decarli, "Massive black hole binaries: dynamical evolution and observational signatures," Advances in Astronomy, vol. 2012, Article ID 940568, 14 pages, 2012.

[7] S. D. Mathur, "The fuzzball proposal for black holes: an elementary review," Fortschritte der Physik, vol. 53, no. 7-8, pp. 793-827, 2005.

[8] A. EL Boukili, M. Nach, K. Bilal, and M. B. Sedra, "Some thermodynamics properties of noncommutative schwarzschild black hole," International Journal of Pure and Applied Physics, vol. 8, no. 3, pp. 143-150, 2012.

[9] A. EL Boukili, M. Nach, and M. B. Sedra, "Hawking radiation Schwarzschild black hole in fuzzy space," International Journal of Basic and Applied Sciences, vol. 2, no. 1, pp. 81-86, 2013.

[10] A. Larranaga and J. M. Tejeiro, "Three dimensional charged black hole inspired by noncommutative geometry," The Abraham Zelmanov Journal, vol. 4, pp. 28-35, 2011.

[11] M. Nach, M. B. Sedra, A. Zemate, and A. EL Boukili, "Electrically charged black hole inspired by 2D fuzzy sphere," Journal of Modern Physics and Applications, vol. 2, no. 1, pp. 31-38, 2013.

[12] L. Modesto and P. Nicolini, "Charged rotating noncommutative black holes," Physical Review D, vol. 82, no. 10, Article ID 104035, 2010.

[13] M. Nach, H. Zaari, and A. El Boukili, "Charged rotating black hole in 2D fuzzy sphere," International Journal of Pure and Applied Physics, vol. 9, no. 1, pp. 21-31, 2013.

[14] Y. S. Myung and M. Yoon, "Regular black hole in three dimensions," European Physical Journal C, vol. 62, no. 2, pp. 405-411, 2009.

[15] M. Nach, A. EL Boukili, M. Brahim Sedra, and A. EL Boukili, "Anti-de-Sitter Schwarzschild black hole in fuzzy space," International Journal of Basic and Applied Sciences, vol. 2, no. 1, pp. 87-90, 2013.

[16] A. EL Boukili, M. Nach, E. L. Boukili, and M. B. Sedra, "Noncommutative BTZ black hole and fuzzy space," International Journal of Basic and Applied Sciences, vol. 2, no. 2, pp. 160-164, 2013.

[17] K. Nozari and B. Fazlpour, "Reissner-Nordström black hole thermodynamics in noncommutative spaces," Acta Physica Polonica B, vol. 39, no. 6, pp. 1363-1374, 2008.

[18] L. Modesto and P. Nicolini, "Charged rotating noncommutative black holes," Physical Review D, vol. 82, no. 10, Article ID 104035 , 2010. 

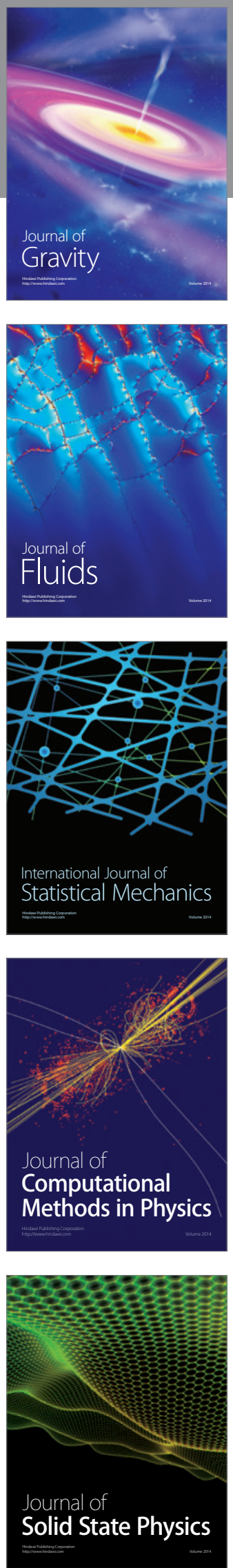

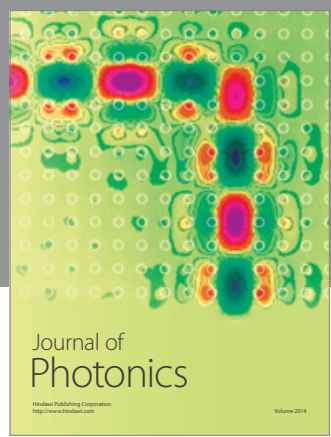

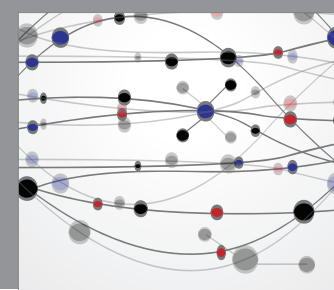

The Scientific World Journal

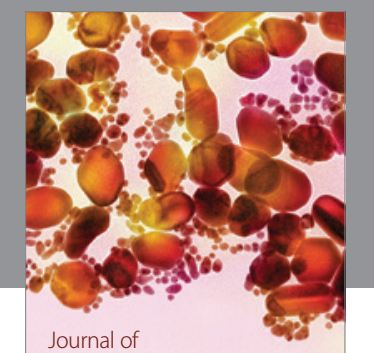

Soft Matter
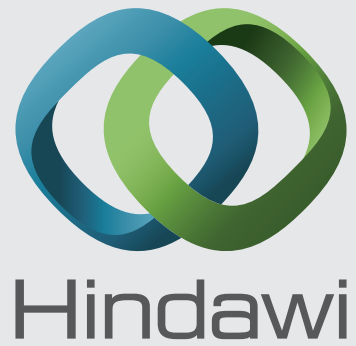

Submit your manuscripts at

http://www.hindawi.com
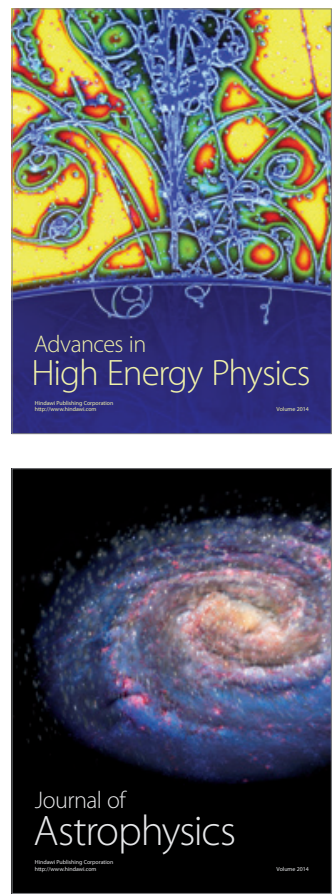
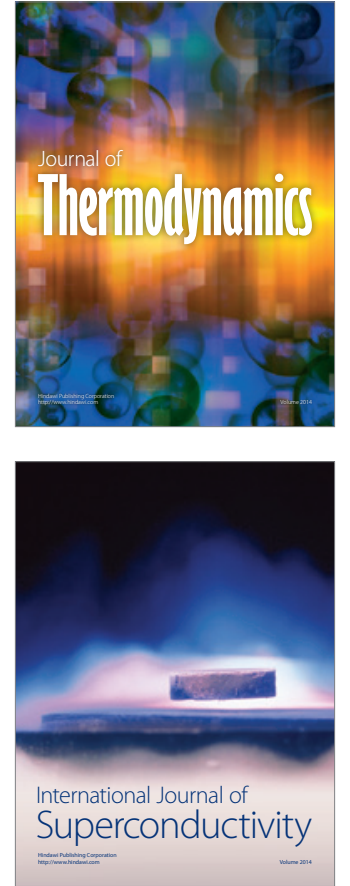
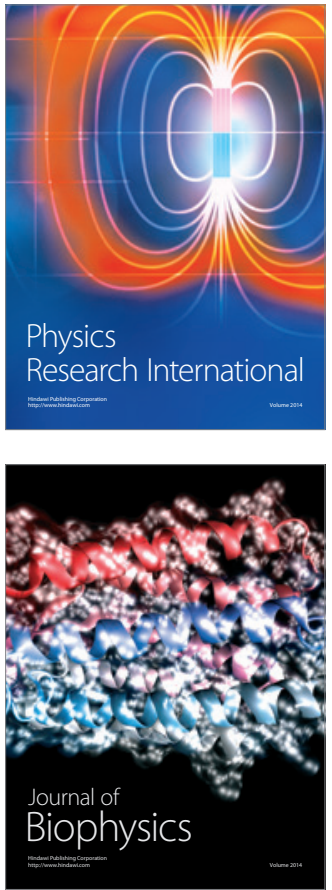
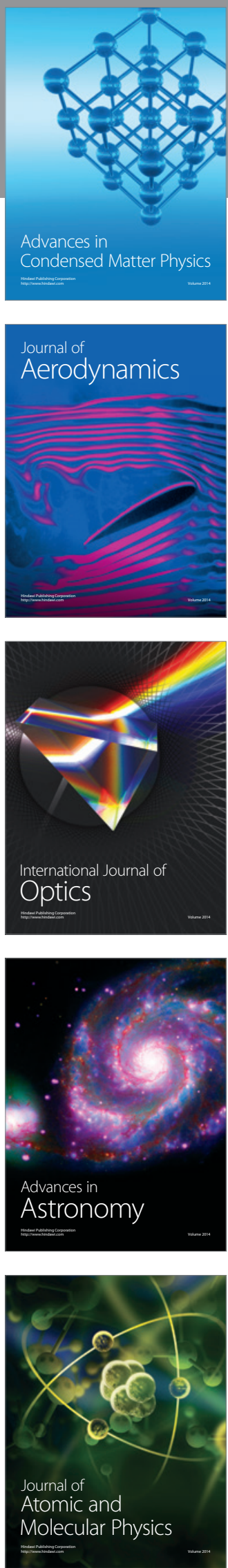